\title{
Decoding information from neural signals recorded using intraneural electrodes: towards the development of a neurocontrolled hand prosthesis
}

\author{
Silvestro Micera, Senior Member IEEE, Luca Citi, Jacopo Rigosa, Jacopo Carpaneto, Stanisa \\ Raspopovic, Giovanni Di Pino, Luca Rossini, Member IEEE, Ken Yoshida, Member IEEE, Luca \\ Denaro, Paolo Dario, Fellow IEEE, Paolo Maria Rossini
}

\begin{abstract}
The possibility of controlling dexterous hand prostheses by using a direct connection with the nervous system is particularly interesting for the significant improvement of the quality of life of patients, which can derive from this achievement. Among the various approaches, peripheral nerve based intra fascicular electrodes are excellent neural interface candidates, representing an excellent compromise between high selectivity and relatively low invasiveness. Moreover, this approach has undergone preliminary testing in human volunteers and has shown promises.

In this manuscript, we investigated whether the use of intrafascicular electrodes can be used to decode multiple sensory and motor information channels with the aim to develop a finite state algorithm that may be employed to control neuroprostheses and neuro-controlled hand prostheses. The results achieved both in animal and human experiments show that the combination of multiple sites recordings and advanced signal processing techniques (such as wavelet denoising and spike sorting algorithms) can be used to identify both sensory stimuli (in animal models) and motor commands (in a human volunteer). These findings have interesting implications, which should be investigated in future experiments.
\end{abstract}

This work was supported in part by the EU within the NEUROBOTICS Integrated Project (IST-FET Project 2003-001917, The fusion of NEUROscience and roBOTICS).

S. Micera is with the Advanced Robotics Technology and Systems Lab, Scuola Superiore Sant'Anna, 56127 Pisa, Italy, the Institute for Automation, Swiss Federal Institute of Technology, Zurich $(\mathrm{CH})$, and with the Rehabilitation Institute and Technology Zurich (RITZ), Zurich (CH) (phone: 39-050-883454; fax +39-050-883497; e-mail: micera@sssup.it).

L. Citi, J. Rigosa1, J. Carpaneto, S. Raspopovic, and P. Dario are with the Advanced Robotics Technology and Systems Lab, Scuola Superiore Sant'Anna, 56127 Pisa, Italy.

G. Di Pino and L. Rossini are with the Biomedical Robotics and Biomicrosystems Lab, Campus Biomedico University, 00128 Rome, Italy.

K. Yoshida is with the Indiana University-Purdue University Indianapolis, 46202 Indianapolis, USA

L. Denaro is with the Dept. of Neurosurgery, Catholic University, 00128 Rome, Italy.

P.M. Rossini is with the Casa di Cura S. Raffaele, and IRCCS S. RaffaelePisana, 00128 Rome, Italy and with the Dept. of Neurology, Campus BioMedico University, 00128 Rome, Italy.
Index Terms-Bionics, bio-robotics, cybernetic hand prostheses, electroneurographic (ENG) signals, hybrid bionic systems, intra-neural interfaces.

\section{INTRODUCTION}

S everal research groups are currently working to develop artificial limbs aimed at restoring or enhancing natural human functions lost/reduced for different reasons (neurological disorders, injuries, amputations). In order to achieve an effective solution, one of the most important requisites is the development of an intuitive interface between the natural and the artificial systems, allowing the user to interact with the artificial system in a simple and natural manner. In recent years, several attempts have been carried out [1-6] to restore this natural link by developing different Hybrid Bionic Systems (HBSs, [5-6]). Among HBSs, active hand prostheses represent an interesting example of the need for natural interfaces between biological and artificial devices. In fact, real usability and comfort of a robotic limb can be accomplished only if the user can feel and control it naturally as if it were part of his/her body. Therefore, a fast, intuitive, bi-directional flow of information between the user's nervous system and the smart robotic device, needs to be established. Presently, this is limited by the interface between biological and mechatronic systems. Several approaches are possible and are currently investigated by different groups as shown in Table 1 and Figure 1.

Among these approaches, Kuiken et al. [12-13] developed a new method based on transferring amputees' residual nerves to other muscles in or near the residual limb (see Figure 1). This approach has the advantage of physiologically correlating the nerve function to the function it is controlling in the prosthesis. Therefore, the user operates in a natural context that is more logical and easier to learn than current EMG-based control paradigms. Moreover, the delivery of sensory feedback via external stimulation seems to be possible both with mechanical and electrical stimuli [3,17-18]. However, the approach seems most suitable for subjects with 
a proximal amputation (shoulder or near axillary level) and requires the use of external devices, for recording and stimulating, without being completely non-invasive.

TABLE I

COMPARISON BETWEEN THE CHARACTERISTICS OF THE DIFFERENT HUMANMACHINE INTERFACES FOR THE CONTROL OF HAND PROSTHESES IN AMPUTEES

\begin{tabular}{|c|c|c|}
\hline Approach & Main Advantages & Main Disadvantages \\
\hline $\begin{array}{l}\text { Surface } \quad \text { EMG } \\
{[7-9]}\end{array}$ & Non-invasive & $\begin{array}{l}\text { Non-natural control } \\
\text { strategies must be } \\
\text { learnt by the subject }\end{array}$ \\
\hline $\begin{array}{l}\text { Implantable } \\
\text { EMG [10-11] }\end{array}$ & $\begin{array}{l}\text { Improved quality of } \\
\text { EMG signals }\end{array}$ & $\begin{array}{l}\text { Non-natural control } \\
\text { strategies must be } \\
\text { learnt by the subject }\end{array}$ \\
\hline $\begin{array}{l}\text { Targeted } \\
\text { reinnervation } \\
{[12-13]}\end{array}$ & $\begin{array}{l}\text { More natural control } \\
\text { strategies, effective } \\
\text { sensory feedback }\end{array}$ & $\begin{array}{l}\text { Requires a surgical } \\
\text { implantation but works } \\
\text { with non-invasive } \\
\text { signals. More suitable } \\
\text { for amputations at the } \\
\text { shoulder level }\end{array}$ \\
\hline $\begin{array}{l}\text { Implantable } \\
\text { cortical } \\
\text { interfaces [14] }\end{array}$ & $\begin{array}{l}\text { Direct connection with } \\
\text { the cortex }\end{array}$ & $\begin{array}{l}\text { Too invasive for the } \\
\text { disability related to } \\
\text { amputation }\end{array}$ \\
\hline $\begin{array}{l}\text { Non-invasive } \\
\text { cortical } \\
\text { interfaces [15] }\end{array}$ & Non-invasive approach & $\begin{array}{l}\text { Limitations in the } \\
\text { number of controllable } \\
\text { degrees of freedom. } \\
\text { Non-natural control } \\
\text { strategies }\end{array}$ \\
\hline $\begin{array}{l}\text { Implantable } \\
\text { peripheral } \\
\text { interfaces } \\
2,4,6,16]\end{array}$ & $\begin{array}{l}\text { Potentially selective and } \\
\text { not very invasive (if } \\
\text { compared with other } \\
\text { implantable solutions) }\end{array}$ & $\begin{array}{l}\text { Limitations in terms of } \\
\text { controllable degrees of } \\
\text { freedom and sensory } \\
\text { feedback not clear }\end{array}$ \\
\hline
\end{tabular}

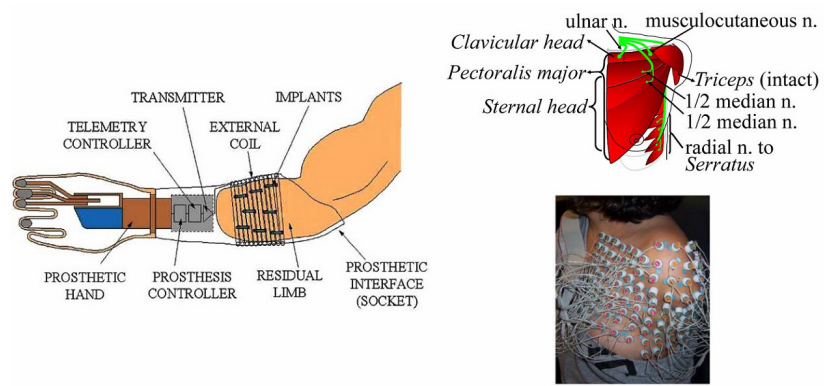

Fig. 1. Schematic (left) of a hand prosthesis based on myoelectric sensors implanted in the muscles of the forearm [11] and schematic (right) of the targeted muscle reinnervation technique and of the high density EMG experimental setup used in unilateral short transhumeral amputee subject [13].

At the same time the use of invasive neural interfaces directly connected to the peripheral nervous system (PNS) is potentially appealing because it may provide, in most cases, an almost "physiological" condition in which efferent and afferent fibers, previously connected with the natural hand, return to their limb/hand control role.

Several invasive PNS interfaces have been developed in the past [4,19-20] (see Figure 2).

Although most devices were originally developed for functional electrical stimulation (FES) in spinal cord injured persons [21-23], they can also be the key component of neurocontrolled hand prostheses. In this case, they are used to record efferent motor signals and to stimulate afferent nerves (i.e., in a complementary way with respect to FES systems) [4]. Among them, Longitudinal intra-fascicular electrodes (LIFEs), intraneural electrodes inserted longitudinally into the nerve tissue [24], are potentially very interesting due to their selectivity and relatively low level of invasiveness. In fact, a recent study showed that the principle of bidirectional control of artificial devices [1-2] was possible in short-term clinical trials with amputees. In particular, it was shown that the subjects were able to control a one-degree of freedom prosthesis by processing efferent neural signals and to receive robust and reliable sensory feedback by stimulating the afferent nerves.

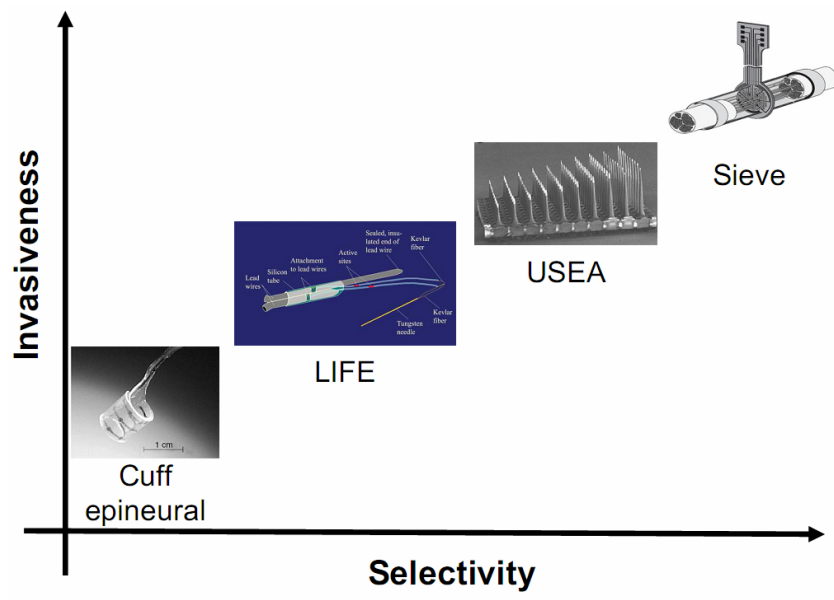

Fig. 2. Different invasive PNS interfaces according to their properties in terms of selectivity and invasiveness.

However, there are some important questions yet to be investigated in order to fully understand the risk/benefits of this approach: (i) whether more advanced processing algorithms can improve the performance of multiple information coding; (ii) whether it is possible to extract motor commands to control more than one degree of freedom from efferent signals recorded from human volunteers.

To address the first question, experiments in the animal model were carried out. The second question was addressed by implanting a new version of LIFEs in a right-handed male (P.P.) who had suffered left arm trans-radial amputation due to a car accident 2 years earlier.

In the next two sections the results achieved in these two cases are given.

\section{EXPERIMENTS IN THE ANIMAL MODEL}

\section{A. Physiological basis}

Extracellular electrodes (e.g., LIFEs) measure the activity of and act upon the population of axons in their immediate vicinity. In general, the interface is non-specific, and the information they measure in the unit activity conveys a mixture of different and time-overlapping data. They can originate from efferent and afferent units. Motor commands can project to different size motor units, or even to different muscle groups. The sensory information set can be varied and 
can originate from kinesthetic, temperature, touch, and pain sensors.

In single-unit recordings, the shape of the action potential is generally believed to carry individual and selective information, with the information coded in the action potential rate and population firing distribution; in other words, the signal-to-noise ratio is remarkably superior. In multi-unit recordings, this information is lost unless it is possible to recover single-unit spike trains from the interleaved spiking activity.

Signals related to different nerve fibers can be identified and extrapolated on the basis of the shape of the unit extracted from the multi-unit recording. The shape of the spike is determined by: the surface area of the membrane (which depends on the axon diameter), the type of fiber (myelinated or not), the orientation of the nodes of Ranvier, the distance between the fiber and the electrode, and the inhomogeneity of the conductivity of the intrafascicular space. Once identified using spike sorting (since different nerve fibers carry different information: e.g., which motor units to activate to perform a given grasp), the spike sorted data can be used to extract specific information, which is carried in the single unit activity, from the mixed population record, thus effectively increasing the resolution of the information that can be extracted from a single electrode. This represents an improvement in the performance of the neural interface and can be used to further reduce invasiveness since fewer electrodes would be required to obtain the needed number of information channels. Spike sorting techniques have been successfully used to process neural activity recorded from cortical interfaces. In the work presented in this section, the applicability of these techniques to peripheral recordings was tested.

\section{B. Thin-film LIFEs}

Thin-film LIFEs (tfLIFE) [25-26] were used in the experiments. These electrodes were developed on a micropatterned polyimide substrate, which was chosen because of its biocompatibility, flexibility and structural properties [27]. The structure (shown in Figure 3) is double sided and has eight evenly spaced recording sites with four sites per side.

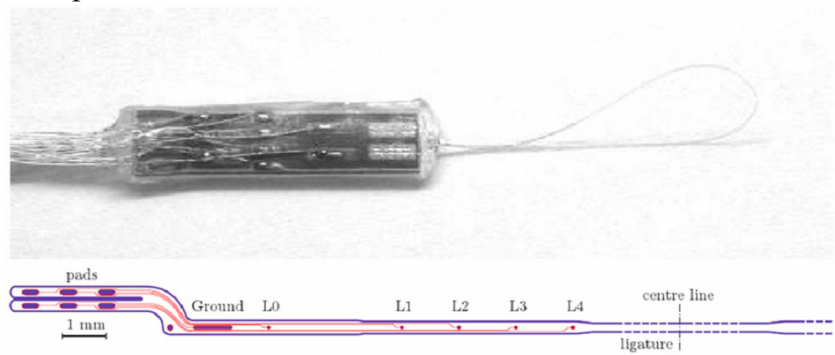

Fig. 3. Picture and unfolded overview of tfLIFE [26]. Total length: $60 \mathrm{~mm}$. Length without pad areas: $50 \mathrm{~mm}$. Each end of the tfLIFE carries a ground electrode (GND), an indifferent recording electrode (L0, R0) and the recording sites (L1-L4, R1-R4).

\section{Protocol of the experiments for classification}

TfLIFEs were implanted in the sciatic nerve of five adult female rabbits. More information can be found in [28]. Various sensory stimuli were applied to the hind limb (i.e., ankle flexion/extension, flexion/extension of one or more toes, and stroking of cutaneous receptive fields) and the elicited signals were recorded using the tfLIFEs. Each stimulus was repeated 4 to 6 times. The complete list of stimuli for each rabbit is provided in Table 2 .

TABLE II

THE DIFFERENT STIMULI APPLIED TO THE ANIMALS. ANIMAL B IS NOT INCLUDED IN THE ANALYSIS BECAUSE IT WAS NOT POSSIBLE TO RECORD ANY SIGNAL FROM THAT ANIMAL. REPRODUCED WITH PERMISSION FROM [28].

\begin{tabular}{cll}
\hline \hline \multirow{2}{*}{ Session } & Stim. Description & Stim. Label \\
& Squeezing of the foot & sqf \\
A & $\begin{array}{l}\text { Ankle flexion } \\
\text { Toe extension } \\
\text { Toe extension combined with ankle flexion }\end{array}$ & $\begin{array}{l}\text { af } \\
\text { te } \\
\text { af_te }\end{array}$ \\
& Stroking of medial plantar area & smpp \\
& Stroking of distal plantar area & sdpp \\
C & Extension of toes $\left(2^{\text {nd }}, 3^{\text {rd }}, 4^{\text {th }}\right)$ & et \\
& Ankle flexion & af \\
\hline \multirow{3}{*}{ D } & Ankle flexion at $90^{\circ}$ & a90 \\
& Release from ankle flexion & a90r \\
& Ankle extension at $175^{\circ}$ & a175 \\
\hline \multirow{4}{*}{ E } & Stroking of paw with ankle at $90^{\circ}$ & sp_a90 \\
& Stroking of paw with ankle at $175^{\circ}$ & sp_a175 \\
& Ext. $2^{\text {nd }}$ and $3^{\text {rd }}$ toes (with ankle neutral) & te_an \\
& Ext. $2^{\text {nd }}$ and $3^{\text {rd }}$ toes (with ankle flexed) & te_af \\
& Flex. $2^{\text {nd }}$ and $3^{\text {rd }}$ toes (with ankle flexed) & tf_af \\
& Flex. $2^{\text {nd }}$ and $3^{\text {rd }}$ toes (with ankle extended) & te_ae \\
\hline
\end{tabular}

For each animal, the recording site with best signal to noise ratio was chosen using the automated procedure described in [28].

A scheme of the general processing and classification approach is shown in Figure 4.

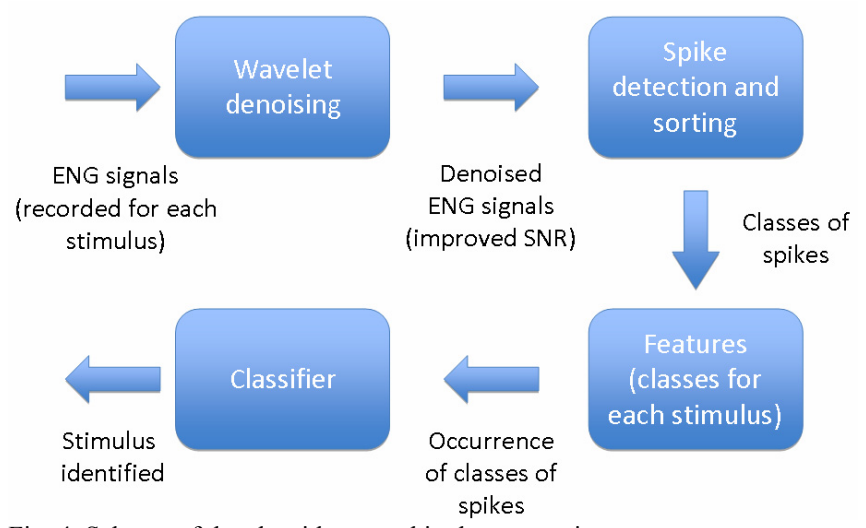

Fig. 4. Scheme of the algorithms used in these experiments

\section{Processing algorithms}

Wavelet denoising (WD) is a set of techniques for removing noise from signals and images [29]. The main idea is to transform the noisy signal into noisy wavelet coefficients in an orthogonal time-frequency domain. In this domain, the coefficients are thresholded to reduce the noise. Finally the 
coefficients are transformed back into the original time domain denoised. A decomposition scheme based on the translation-invariant wavelet transform [30] was used.

After this preliminary step, an algorithm for spike detection and sorting was applied to the data. The spike sorting process consisted of two phases. During the first phase, a subset of the recordings (training set) was used to individuate recurring spike shapes and to build a dictionary of spike templates. During the second phase, the remaining part of the recordings (test set) was scanned looking for occurrences of the spike templates previously identified. The wavelet denoising and the spike sorting algorithms allowed the extraction of specific spike waveforms as those shown in Figure 5.

\section{E. Classification Algorithms}

The different epochs related to the different classes (i.e., the types of stimuli from Table 2) were labeled and used as examples to train the classifier or to test its generalization skills. For each example, the feature vector was composed of the ratios between the number of spikes matching each template and the total number of spikes in the epoch [28]. Therefore, the absolute spike rates were not used, but rather the relative spike rates of each waveform w.r.t. the others (Srt). This should prevent classification of the epochs based on the "quantity of activity" and favor the use of the "quality of activity", intended in terms of different waveforms for different stimuli. The reason for this approach has been previously described. In order to infer the type of stimulus applied during a given epoch, a classifier based on support vector machines (SVMs) [31] was used.

\section{F. Validation scheme}

A validation scheme was developed by using a single example per class (stimulus type) as the test set (i.e., never used for tuning any parameter, creating spike templates, or training the machine). The remaining examples were used as the training set. For example, if a given session had 9 epochs for each of the five different types of stimuli, the test set would consist of 5 epochs while the training set of 40 epochs.

In order to obtain an average performance with small confidence intervals, a random subsampling cross-validation was used. The following procedure was repeated 5000 times: (i) build the test set by randomly selecting one epoch for each stimulus type (e.g., one set of 5 epochs among $9^{5}$ combinations for the example above); (ii) build the training set by selecting the remaining epochs; (iii) run the template creation phase of the spike sort algorithm on the training set; (iv) run the template matching phase of the spike sort algorithm on the whole set; (v) use the features from the training set to train a SVM machine; (vi) make the SVM machine predict the stimuli type for the test set and compare it with the ground truth.

\section{G. Results}

The performance of the system for each experimental session is reported in Table 3. The first column (WD/Srt) shows the performance in terms of percentage of correct classifications and channel capacity, for the approach presented here. For comparison, the last column reports the results of a typical ENG approach (FIR/RBI) using a bandpass filter (FIR) between 700 and $2000 \mathrm{~Hz}$ and a rectification and bin-integration (RBI) evaluated as the mean over the epoch of the RBI computed on 50ms windows. The other columns present the performance of hybrid configurations.

TABLE III

PERFORMANCE OF SESSIONS WITH 4 (A, C, E) OR 5 (D) STIMULI IN TERMS OF PERCENTAGE OF CORRECT CLASSIFICATIONS (PC [\%]) AND CHANNEL CAPACITY (C [BIT/SYMB]). TWO DIFFERENT PREPROCESSING METHODS - WAVELET DENOISING (WD) AND BANDPASS FILTER (FIR) - AND TWO DIFFERENT FEATURE CREATION METHODS - RELATIVE SPIKE ACTIVITY (SRT) AND RECTIFICATION AND BIN-INTEGRATION (RBI) - WERE COMPARED. REPRODUCED WITH PERMISSION FROM [28].

\begin{tabular}{cccccc} 
Session & Measure & WD/Srt & FIR/Srt & WD/RBI & FIR/RBI \\
& & & & & \\
\hline \multirow{2}{*}{$\mathrm{A}$} & PC & 92.6 & 66.7 & 68.6 & 52.8 \\
& $\mathrm{C}$ & 1.59 & 0.87 & 1.01 & 0.84 \\
\hline \multirow{2}{*}{$\mathrm{C}$} & PC & 99.1 & 95.2 & 88.8 & 69.0 \\
& $\mathrm{C}$ & 1.92 & 1.72 & 1.50 & 1.28 \\
\hline \multirow{2}{*}{$\mathrm{D}$} & PC & 94.5 & 80.4 & 76.0 & 66.5 \\
& $\mathrm{C}$ & 2.01 & 1.64 & 1.61 & 1.54 \\
\hline \multirow{2}{*}{$\mathrm{E}$} & PC & 90.1 & 60.7 & 92.6 & 89.1 \\
& $\mathrm{C}$ & 1.51 & 0.64 & 1.71 & 1.51 \\
\hline
\end{tabular}

These results confirm that more advanced processing algorithms can improve performance. The proposed approach does so by trying to un-mix the combined activity in multiunit recordings and recover the information carried by the single (or smaller groups of) nerve fibers.

Figure 5 is an example that helps to illustrate how the system works internally. It contains two minute data from session D. The left panel shows 5 of the 17 templates that were found. Templates \#3 and \#4 were considered together because they have similar properties and behaviors. Templates $\# 9$ and \#10 were similarly combined. The abscissa of the right panel is time, and shows time epochs delineated by the segments separated by dotted lines where different types of stimuli were applied to the paw. Each row shows the frequency of template "hits" for a given template, with the first row corresponding to template $\# 1$, the $2^{\text {nd }}$ row corresponding to template $\# 3$ and $\# 4$, and the $3^{\text {rd }}$ row corresponding to template $\# 9$ and $\# 10$. The relative "hit" rate of template \#1 is maximum during ankle flexion (a90), while the maximum rate for templates \#3 and \#4 is maximum during release from flexion (a90r) and for templates $\# 9$ and \#10 is maximum during extension (a175). The example illustrates that 3 different types or channels of information are extracted through the use of spike sorting from a single electrode channel.

\section{CASE STUdy WITH AN AMPUTEE}

\section{A. Subject and methods}

P.P. was a 26 year-old male who had suffered left arm amputation two years earlier. Phantom awareness and 
presence
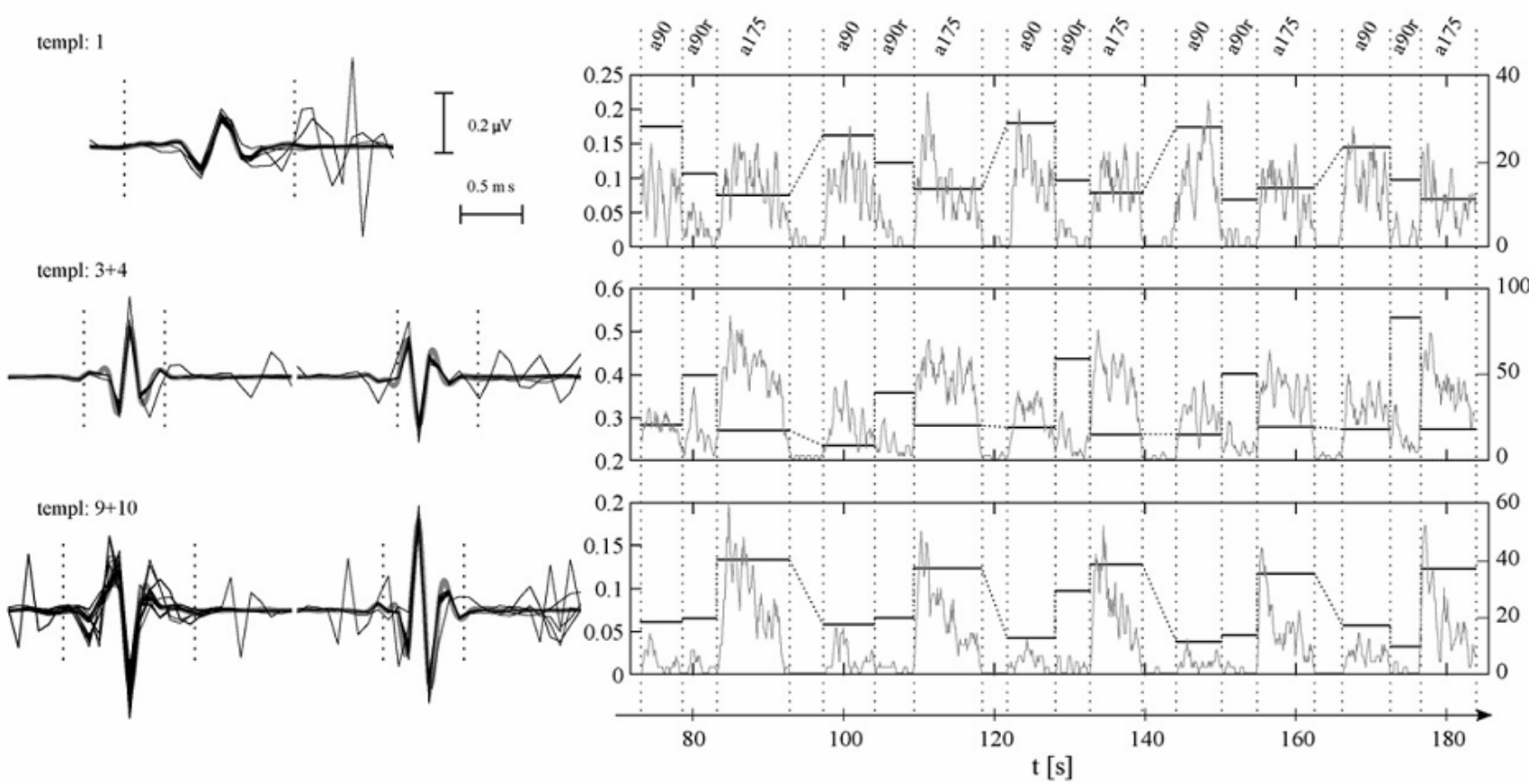

Fig. 5. Analysis of how different "spike waveforms" could help the detection of specific stimuli (a90, a90r, a175, see Table 1). On the left, some of the spike waveforms found are given. The thick gray lines represent the waveforms identified during the "template creation" phase and their support is delimited in the plots by the dotted vertical bars. The thin black lines represent some of the spikes identified as belonging to that specific template. On the right, the black lines (scales on the left) represent the ratio fi between the occurrence of the correspondent "spike waveform" shown on the left and the total spikes detected for each epoch. The gray lines (scales on the right) are the absolute spike rate of the corresponding spike waveforms evaluated on 1-s sliding windows. Reproduced with permission from [28]

of Phantom Limb Syndrome were evaluated pre-surgically and were followed up at the end of the training period and 3 months after LIFEs removal. Following epineural microdissection, two tf-LIFE4s separated by $3 \mathrm{~cm}$, were inserted in ulnar and median nerves $45^{\circ}$ obliquely to assure stability and to increase the probability of intercepting nerve fibers. The distal handle of the electrode was anchored to the epineurium. Four weeks later, tf-LIFE4s were removed as required by the European Health Authorities. P.P. worked on the project 4-6 hours/day for 6 days/weekly and did not report any complication during the 3 month follow-up period.

\section{A. Experimental protocol}

Electroneurographic (ENG) and electromyographic (EMG, biceps/triceps, surface belly-tendon recordings) signals were recorded. P.P. was trained to dispatch three individual movement commands to the missing hand : (i) power grip; (ii) pinch grip; (iii) flexion of the little finger. Each movement was identified by an individual trigger that was used during signal processing in order to discriminate voluntary activities only in the expected parts of the signal. Imagined movements consisted in alternating moments of immobility (2-4 s) with hand movements (around $1 \mathrm{sec}$ ), and were synchronized with the recording system. Signals both from the tf-LIFEs and EMG electrodes were simultaneously recorded, sampled at $48 \mathrm{kHz}$, and mean rectified in data-windows of 1000 samples. In order to reduce cognitive burden and to help P.P. to dispatch selective motor orders, two different recording phases were designed: (1) P.P. was asked to imagine only one class of movement (power grip) during the first two weeks and then (2) all three classes during the final week.

Immediate off-line signal evaluation identified the best contacts for repeatability and signal-to-noise ratio. After the best candidate-channels had been identified, P.P. was asked to control their online modulation - this being the most efficient training protocol - and to maintain EMG activity as low as possible.

More detailed information about the protocol and the clinical results can be found in [32].

\section{B. Processing and classification algorithms}

The algorithms presented in the previous section for animal experiments were also used in the experiments with P.P. Figure 6 provides some examples of classes extracted from the ENG efferent signals.

However, the possibility of using signals simultaneously acquired from more than one channel for classification was also considered. Wavelet denoising allowed the identification of several classes of spikes. The different features were then used as inputs to classify grip types and rest. In Table 4 , the best performance achieved with the best combination of tfLIFE channels is shown for the classification of rest plus one, two, or three grip types.

Moreover, in order to understand the importance of 
multi-channel electrodes, classification performance was calculated for the different number of channels.

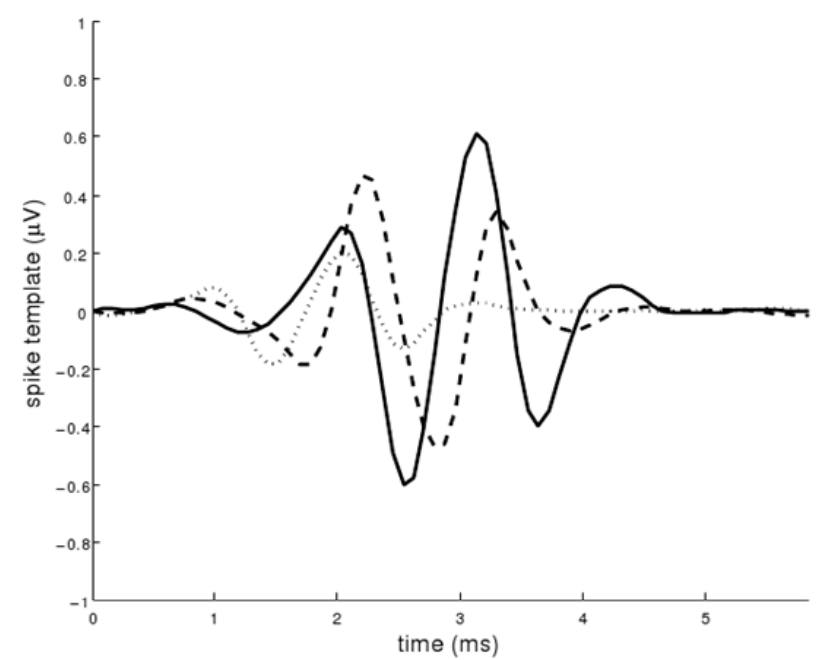

Fig. 6. Examples of templates of spikes extracted from efferent ENG signals

The results are shown in Figure 7 for two different classification methods: (1) discrimination of four classes simultaneously (rest, three grip types); (2) hierarchical discrimination (rest versus activities and then selection of grip type). The results indicate that the use of several channels can increase performance not only due to the increased amount of information but also due to increased flexibility. In fact, by using hierarchical discrimination, different channels and classes can be used for the two steps and performance is increased with respect to the nonhierarchical classification approach.

TABLE IV

THE BEST PERFORMANCE (DAY 30) FOR THE CLASSIFICATION PERCENTAGE (PC [\%]) FOR ONE, TWO, AND THREE CLASSES AGAINST REST.

\begin{tabular}{ccc}
\hline \# of classes & Class(es) description & PC \\
\hline 3 classes (and rest) & little, fist, and pich & 85 \\
\hline 2 classes (and rest) & little, fist & 92 \\
\hline 2 classes (and rest) & little, pinch & 90 \\
\hline 2 classes (and rest) & fist, pinch & 87 \\
\hline 1class (and rest) & little & 93 \\
\hline 1class (and rest) & fist & 95 \\
\hline 1class (and rest) & pinch & 100 \\
\hline
\end{tabular}

\section{DISCUSSION}

The aim of our study was to increase knowledge on the possibilities and limits of computerized analysis of signals emitted by peripheral fibers during in general by means of intrafascicular electrodes and in particular during tf-LIFE simultaneous recordings from multiple nerve sites. In particular, our assumption was that different neural commands can generate different classes of spikes recorded with the LIFEs and that these classes can be used to discriminate different sensory or motor information.

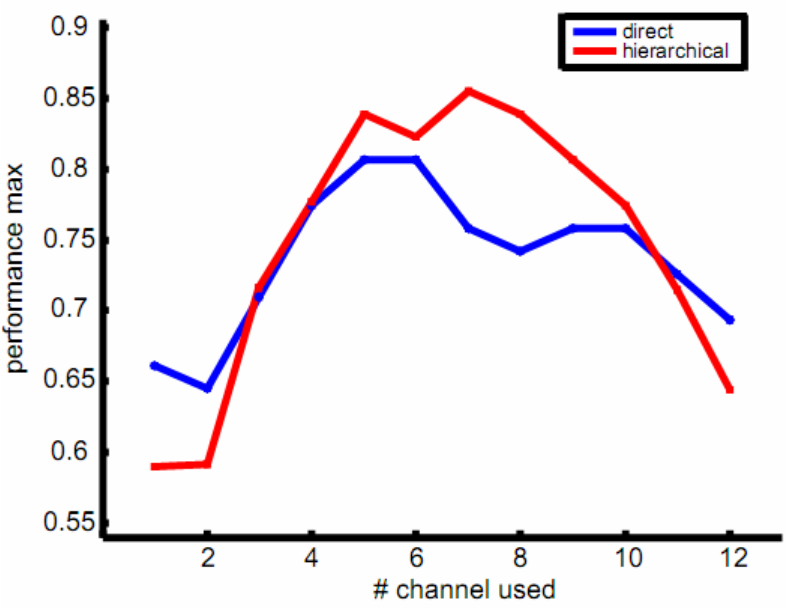

Fig. 7. Maximum performances obtained using direct and hierarchical classification are shown as a function of the number of channel for Day 30 signals.

The experiments with animal models showed that the combination of wavelet denoising and spike sorting techniques can increase the amount of information extracted from ENG signals recorded with intraneural electrodes. This approach could be used in the future to develop closed-loop controlled neuroprostheses based on natural sensory information.

The same processing approach was then used to analyze the ENG efferent signals recorded during an experiment with a human volunteer. In this case, the results in terms of classification of motor tasks showed that a state control algorithm can be implemented. Three different hand movements can be identified with quite good performance. Even more interesting was P.P.'s ability to improve his performance during the trials. This shows that the performance of chronic implantations could possibly improve.

Unfortunately, given the time limitations of our experiments it was not possible to increase the number of grip types that subjects were asked to dispatch to the missing hand. However, it is now clear that several movements can be classified and that probably they can easily be more than three as in our study. This goal can be easily and rapidly achieved by the subject due to the fact that he has nearly nothing to learn, since the emitted signal follow about the same programming/execution parameters as in the natural life when using an intact hand. Performance can also be improved by using more intraneural channels. This is due to the intrinsic blindness of the implantation procedure: a greater number of channels can increase the probability of receiving more useful signals for classification. Another possibility is to embed micro-actuators [33-34] in the structure of the neural interface to move the different electrical contacts in the nerve (see Figure 8). 
(a)

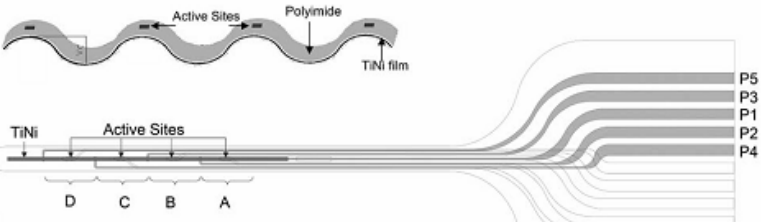

(b)

(c)

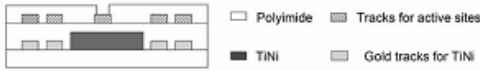

Fig. 8. Corrugated profile ( $\mathrm{Vc}=$ height of the peaks), (b) drawing of the multiactuation layout, and (c) cross section of an actuated intraneural electrode [34].

The results showed in this manuscript indicate that some specific "states" (i.e., grip types) can be decoded from intraneural ENG signals. This "high-level" finite state approach has been implemented with very good results in EMG-controlled prostheses [5, 13, 35]. It is achieved by combining information on the grip together with fixed parameters embedded in the robotic controller (e.g., on the pre-shaping phase of the hand before grasping) and selected by trial and error. This kind of information could then be used as desired state to be achieved by a low-level control algorithm (from very simple PID controllers to complex force-position algorithms). The level of "shared-control" between the user's brain and the robotic controller can be modified according to the performance of the neurocontrolled hand prosthesis. In particular, the extraction of more reliable information can reduce the importance of the robotic controller, by relaying more on the user's intentions and during the training phase.

As already shown for the decoding and control of upper limb movements using cortical signals [36], this "sharedcontrol" approach can significantly increase the performance and the effectiveness of the overall system. The information from the artificial sensors embedded in the hand can be used to define different low-level control strategies (for example, avoid slippage or achieve obstacle avoidance and accurate grasping) and to check the correspondence between the actual desired activity and the current task of the robotic system. The shared-control paradigm can be introduced in the form of the artificial field potentials described in [37]. In this case the robot moves in fields of forces generated by the user (through the decoding algorithms,) and by the different sensors (i.e., the total potentials will be $\left.\mathrm{U}_{\text {decoding }}+\mathrm{U}_{\text {sensors }}\right)$. When, for example, one or more sensors placed in the artificial hand detects the proximity of an object, it causes a sensor-based field potential, which may have local attractive or repulsive regions depending on the control laws of the individual sensors. These shared-control strategies can be continuously updated on-line according to the information provided, for example, by a reinforcement learning algorithm. This could be done by learning the parameterisation of a specific additional force field to be added (i.e., the total potentials will be
$\mathrm{U}_{\text {decoding }}+\mathrm{U}_{\text {sensors }}+\mathrm{U}_{\text {learning }}$ ). The learning process could affect not only the user (through invasive or non-invasive sensory feedback) but also the artificial components of the neurocontrolled hand prosthesis. Similar examples of colearning between the user and the decoding algorithm have been exploited for cortical neuroprostheses with very promising results [38].

All these preliminary results need to be confirmed during more extensive chronic experiments both in animals and selected human volunteers. It would be particularly interesting to understand the maximum amount of information, which can be extracted, and the robustness of this approach in terms of need for frequent recalibration of the algorithms used for classification.

\section{REFERENCES}

[1] G.S. Dhillon, T.B. Krüger, J.S. Sandhu, and K.W. Horch," Effects of short-term training on sensory and motor function in severed nerves of long-term human amputees," J Neurophysiol. vol. 5, pp. 2625-33, 2005.

[2] G.S. Dhillon and K.W. Horch, "Direct neural sensory feedback and control of a prosthetic arm", IEEE Trans Neural Syst Rehabil Eng., vol 13 pp. $468-72,2005$.

[3] N.G. Hatsopoulos and J.P. Donoghue, "The science of neural interface systems", Annu Rev Neurosci, vol 32, pp. 249-66, 2009.

[4] X. Navarro, T.B. Krueger, N. Lago, S. Micera, T. Stieglitz and P. Dario, "A critical review of interfaces with the peripheral nervous system for the control of neuroprostheses and hybrid bionic systems," J Peripher Nerv Syst, vol. 10, pp. 229-258, 2005.

[5] S.Micera, M.C. Carrozza, L. Beccai, F. Vecchi, and P. Dario, "Hybrid Bionic Systems for the Replacement of Hand Function," Proc IEEE, vol. 94, pp. 1752-1762, 2006

[6] S. Micera, X. Navarro, J. Carpaneto, L. Citi, O.Tonet, P. Rossini, M. Carrozza, K. Hoffmann, M.Vivò, K. Yoshida, and P. Dario, "On the use of longitudinal intrafascicular peripheral interfaces for the control of cybernetic hand prostheses in amputees," IEEE Trans Neural Syst Rehabil Eng, vol. 16, pp. 453-472, 2008.

[7] M. Zecca, S. Micera, M.C. Carrozza and P. Dario, "Control of multifunctional prosthetic hands by processing the electromyographic signal," Crit Rev Biomed Eng, vol. 30, pp. 459-485, 2002.

[8] M.A. Oskoei and H. Hu, "Support Vector Machine-Based Classification Scheme for Myoelectric Control Applied to Upper Limb," IEEE Trans Biomed Eng, vol. 55, pp.1956-65, 2008.

[9] K. Englehart and B. Hudgins, "A robust, real-time control scheme for multifunction myoelectric control," IEEE Trans Biomed Eng, vol. 50, pp.848-854, 2003.

[10] T.R. Farrell and R.F. Weir, "The optimal controller delay for myoelectric prostheses," IEEE Trans Neural Syst Rehabil Eng, vol. 15, pp. 111-118, 2007

[11] R.F. Weir, P.R. Troyk, G.A. DeMichele, D.A. Kerns, J.F. Schorsch, and H. Maas, "Implantable myoelectric sensors (IMESs) for intramuscular electromyogram recording," IEEE Trans Biomed Eng, vol. 56, pp.159-171, 2009.

[12] T. Kuiken, L. Miller, R. Lipschutz, B. Lock, K. Stubblefield, P. Marasco, P. Zhou and G Dumanian, "Targeted reinnervation for enhanced prosthetic arm function in a woman with a proximal amputation: a case study," The Lancet, vol. 369 pp. 371-380, 2007.

[13] H. Huang, P. Zhou, G. Li, and T. A. Kuiken, "An analysis of EMG electrode configuration for targeted muscle reinnervation based neural machine interface," IEEE Trans Neural Syst Rehabil Eng, vol. 16, pp. 37-45, 2008.

[14] L.R. Hochberg, M.D. Serruya, M.G. Friehs, J.A. Mukand, M. Saleh, A. H. Caplan, A. Branner, D. Chen, R.D. Penn and J.P. Donoghue, "Neuronal ensemble control of prosthetic devices by a human with tetraplegia," Nature, vol. 442 pp.164-71, 2006.

[15] J. Wolpaw and J. McFarland "Control of a two-dimensional movement signal by a noninvasive brain-computer interface in humans," Pnas,vol.101 pp.17849-54, 2004. 
[16] X. Jia , M.A. Koenig, X. Zhang, J. Zhang, T. Chen and Z. Chen, "Residual motor signal in long-term human severed peripheral nerves and feasibility of neural signal-controlled artificial limb," J Hand Surg [Am], vol.32 pp.657-66, 2007.

[17] L.G. Cohen , S. Bandinelli , T.W. Findley and M. Hallett, "Motor reorganization after upper limb amputation in man. A study with focal magnetic stimulation," Brain vol. 114, pp. 615-27, 1991

[18] K. Yoshida and K. Horch, "Selective stimulation of peripheral nerve fibers using dual intrafascicular electrodes," IEEE Trans Biomed Eng., vol. 40 pp. 492-4,1993

[19] M. Haugland, A. Lickel, J. Haase, and T. Sinkjær, "Control of FES thumb force using slip information obtained from the cutaneous Electroneurogram in Quadriplegic Man," IEEE Trans Neural Syst Rehabil Eng, vol. 7, pp. 215-227, 1999.

[20] R.B. Stein, D. Charles, J.A. Hoffer, J. Arsenault, L.A. Davis, S. Moorman and B. Moss, "New approaches for the control of powered prostheses particularly by high-level amputees," Bull Prosthet Res, vol. 10, pp. 51-62, 1980.

[21] D. Guiraud, T. Stieglitz, K.P. Koch, J.L. Divoux and P. Rabischong, "An implantable neuroprosthesis for standing and walking in paraplegia: 5-year patient follow-up,” J Neural Eng, vol. 3, pp. 268275, 2006.

[22] M. Haugland and J. Hoffer, "Slip information provided by nerve cuff signals: application in closed-loop control of functional electrical stimulation," IEEE Trans Rehabil Eng, vol. 2, pp. 29-36, 1994.

[23] A. Inmann and M. Haugland, "Implementation of natural sensory feedback in a portable control system for a hand grasp neuroprosthesis," Med Eng Phys, vol. 26, pp. 449-458, 2004.

[24] S.M. Lawrence, G.S. Dhillon, and K.W. Horch, "Fabrication and characteristics of an implantable, polymer-based, intrafascicular electrode," J Neurosci Methods. vol. 131, pp.9-26, 2003.

[25] K. Yoshida, K. Hennings, and S. Kammer, "Acute performance of the thin-film longitudinal intra-fascicular electrode", in First IEEE/RASEMBS International BioRob Conference, Pisa, p.296-300, 2006.

[26] K.P. Hoffmann and K.P. Kock, "Final report on design consideration of tLIFE2," Tech Rep IBMT, 2005.

[27] F.G. Zeng "Trends in cochlear implants," Trends Amplif vol. 8 pp.134,2004

[28] L. Citi, J. Carpaneto, K. Yoshida, K.P. Hoffmann, K.P. Koch, P. Dario, and S. Micera, "On the use of wavelet denoising and spike sorting techniques to process electroneurographic signals recorded using intraneural electrodes", J Neurosci Methods vol.172 pp.294302, 2008

[29] D.B. Percival and A.T. Walden, "Wavelet Methods for Time Series Analysis," Cambridge University Press, 2000

[30] R.R. Coifman and D.L. Donoho "Translation-invariant de-noising," in: Wavelets and statistics. Springer-Verlag; pp. 125-50; 1995

[31] C. Cortes and V. Vapnik, "Support-Vector Networks," Machine Learning, 20, 1995

[32] P.M. Rossini, S. Micera, A. Benvenuto, J. Carpaneto, G. Cavallo, L. Citi, C. Cipriani, L. Denaro, V. Denaro, G. Di Pino, F. Ferreri, E. Guglielmelli, K.P. Hoffmann, S. Raspopovic, J. Rigosa, L. Rossini, M. Tombini, and P. Dario, "Double nerve intraneural interface implant on a human amputee for robotic hand control," submitted, 2009.

[33] S. Bossi, A. Menciassi, K. P. Koch, K. P. Hoffmann, K. Yoshida, P. Dario, and S. Micera, "Shape Memory Alloy Microactuation of tfLIFEs: Preliminary Results," IEEE Trans Biomed Eng, vol. 54, pp. $1115-1120,2007$.

[34] S. Bossi, S. Kammer, T. Dörge, A. Menciassi, K. P. Hoffmann, and S. Micera, "An Implantable Microactuated Intrafascicular Electrode for Peripheral Nerves," IEEE Trans Biomed Eng, vol. 56, pp. 27012706, 2009

[35] C. Cipriani, F. Zaccone, S. Micera, M.C. Carrozza, "On the shared control of an EMG controlled hand prosthesis: analysis of userprosthesis interaction," IEEE Trans Robotics, vol. 24, no. 1, pp. 170184, 2008.

[36] H.K. Kim, S.J. Biggs, D.W. Schloerb, J.M. Carmena, M.A. Lebedev, M.A. Nicolelis, and M.A. Srinivasan, "Continuous shared control for stabilizing reaching and grasping with brain-machine interfaces," IEEE Trans Biomed Eng, vol. 53, pp. 1164-1173, 2006.

[37] O. Kathib, "Real-time obstacle avoidance for manipulators and mobile robots," Int J Robot Res, vol. 5, pp.90-98, 1986.
[38] J. DiGiovanna, B. Mahmoudi, J. Fortes, J.C. Principe, and J.C. Sanchez, "Coadaptive brain-machine interface via reinforcement learning,” IEEE Trans Biomed Eng, vol. 56, pp.54-64, 2009. 